\title{
Extrinsic electromagnetic fields, low frequency (phonon) vibrations, and control of cell function: a non-linear resonance system
}

\author{
Glen A. Gordon \\ Electromagnetic Research and Education Foundation, PO BOX 124, Port Gamble, WA 98364. Correspondence should be addressed to Glen A. Gordon (drgor- \\ don@emref.com), Tel.: (360) 297-6858. \\ Received August 21, 2008; revised September 10, 2008; accepted September 10, 2008
}

\begin{abstract}
Chou and Chen's report in the 1970s suggested conformational protein adaptation (CPA) might be influenced by low frequency phonons acting as "a possible information system". This report proposes the universal force of electromagnetism initiates the phonon system they cited as it perturbs paramagnetic/diamagnetic dampers within the protein matrix to produce a quantized low frequency phonon signal series. (http://www.phy.ilstu.edu/ ren/phononsims/page3 .html) The signal series is iteratively processed by the protein beta subunit, the system, to position the alpha subunit, the outcome, a classic non-linear resonance system resulting in conformational protein adaptation (CPA).

CPA "priming" enables a secondary ATP/redox driven power system to complete cell activity. The evolutionary appearance of these two systems reflects their hierarchy: 1) a low energy phonon driven information control circuit governed by principles of physics that, along with proteins, may have preceded planet earth, and 2), an ATP/redox power completion circuit directed by principles of chemistry that evolved in living systems 1 billion or more years after earth formed.
\end{abstract}

Keywords: Electromagnetic fields-forcer; Paramagnetic/diamagnetic oscillators-damper; Phonon resonance-signal series; Protein iteration-system; Conformational adaptation-outcome

\section{INTRODUCTION}

Following the "big bang" and earlier formation of paramagnetic hydrogen, star collapse largely completed the atomic chart with three forms of electromagnetic responsive elements, i.e. paramagnetic, diamagnetic, and ferromagnetic; later, atmospheric damping of electromagnetic discharges from lightning and solar sources evolved The
Schumann Resonance(SR). As life evolved on earth the SR was the primary forcer that caused bond length oscillations in paramagnetic/diamagnetic constructs (PDCs) that proteins strategically assembled to initiate and route the resultant quantized signal series. In forcing this signal series that was native to the protein matrix, the SR controlled conformational protein adaptation (CPA) in DNA, protein enzymes, and membrane proteins. Hawking noted in A Brief History of Time, "the universal force of electromagnetism controls all biological response", applicable here just as in Pauling's more classical derivation (Nobel lecture, 1954). Diurnal fluctuations shared by living systems and The SR constitute epochal evidence that native phonon vibrations are shared among proteins to control cell function throughout the hierarchy of living systems. For those who would assign diurnal function to light and dark a review [48] of this atmospheric influence is suggested. This phonon system, symbiotically shared as proteins assembled, and later called a meridian, was functioning nearly 2 billion years before the first nerve network evolved in Cnidarians. (http://trc.ucdavis.edu/biosci10v/bis10v/week10/07nervev olution.) "Lacking a connection to the central nervous system" may not be a valid criticism of the meridian concept.

Information Control Circuit:

The low frequency phonon control circuit is a classic non-linear resonance system that involves iterative processing of this quantized acoustic series by beta sub-units in enzymes, DNA, and membrane proteins, i.e. the system, to conformationally adapt the alpha sub-unit, the outcome. With appropriate "energy coupling" facilitated, ATP/redox power completion dynamics shuttle ions and substrate along metabolic pathways, but only after CPA enables that. When investigated as a focus of life science or clinical research, these completion dynamics should be recognized as a separate rote activity for if activated without informed conformational protein adaptation the result would be chaotic or failed cell function.

While subthreshold in themselves, appropriately damped quantized phonon energy conducts heat and sound through proteins as native low frequency (phonon) vibrations to achieve resonance with harmonics from other 
strategically assembled paramagnetic/diamagnetic constructs (PDCs) within the protein thus enhancing signal intensity several magnitudes [35]. DNA, and other proteins, given their sophisticated capacity to selectively extract harmonics, use these signals in combination with other "noise", i.e. stochastic resonance, to enhance protein function $[6,18,44]$. This selective matching indicates a sophisticated routing capacity in proteins $[33,34]$ which is proposed here to be a co-activity of PDCs. An area of investigative interest in this regard may be the magneto-hydrodynamic effects described by Alfven (Nobel lecture, 1970) and a recent report on Hall effects [50] in phonons

This initiating/routing phonon information system promises market disruptive technologies that could dramatically enhance homeostasis as a system undergoes ischemia-reperfusion injury or other insult [12]. Extant literature [24, 31, 38] suggests significant potential for designed electromagnetic pulsed therapies (DEPTH), the technology to replace classes of drugs and numerous surgeries within the decade.

\section{HISTORY}

The Flexner Report in 1910 declared the universal force of electromagnetism "irregular science" and it was purged from medical curricula in the United States forty-five years after Maxwell defined it; a similar effort in Europe was unsuccessful. 1900 and "The New Era of Science" saw electromagnetism eventually characterized as "controlling all chemical reactions, including life itself" [25]. Considering this universal force and its influence on chemical reactions, it is inexplicable that clinical, biological, and basic science interest in electromagnetism, PDCs, and their relation to cell function languished until MRI stimulated interest in the late $20^{\text {th }}$ century.

\section{NORMAL MODE (ELEMENTARY) PHO- NON VIBRATIONS}

Kuo-Chen Chou developed an iconic archive on the importance of low frequency phonon vibrational activity in proteins following his original insight [17]. He considered low frequency vibrations (phonons) in proteins including DNA implicit to their biological function $[13,14,15,16]$. In citing the "overwhelming production of phonons by native low frequency vibrations", and, "the importance of low frequency vibrations in biologic function from the viewpoint of both thermodynamic and molecular dynamics", he alluded to the conformational adaptation of proteins and control of cell function in response to these native low frequency vibrations as, "an intriguing concept".

W. Ross Adey accumulated an archive [1, 2, 3] on non-linear EMF cellular effects and intrinsic communication systems between cells that he considered, "a general biologic property”. Adey's early soliton concept [36] was later abandoned by his co-author [37].

All constituent elements and amino acids function as PDC dampers within a protein lattice where, if the EM field is appropriate, an elementary or "native" mode pho- non vibration results that is compatible with individual protein design and length. Given this as the means for generating phonons, Kriegl et als [33] report additionally suggests a routing effect exists in biological systems, which prompts the question, "Does electromagnetism influence PDCs to generate phonons and direct their routing as well?" Sophisticated phonon initiation and routing directs conformational protein adaptation to prime redox reactions [52], calcium channel activity, and bioenergetics [17]; up-regulating DNA [12, 49] and enhancing enzyme activity [55] as iterative outcomes are proposed as logical conclusions in other reports,

Curie's magnetic moment (M) of a paramagnetic species, which represents the sum of EMF directed dipole alignment (B), and Brownian vibrational activity (T), is the energy transduced into the protein lattice with sophisticated forward and reverse options [34, 27] to remote sites $[6,18,29,44]$. When an EM field is damped to create a native (elementary) vibrational activity it assures homeostatic CPA as the system experiences challenge.

Johnson et al. [30] noted "unambiguous" evidence for PDC presence in Fe/enzyme models, and Ubbink et al. [52] reported that PDC forces directed conformational protein changes that resulted in apposition of distant protein redox sites and ensuing reaction, redox chemistry controlled by principles of physics.

"Stochastic resonance" is the ability of proteins to guide appropriate harmonics extracted from "noise", to sum with sub-threshold phonon harmonics, another highly evolved adaptive response to enhance sub-threshold native or elementary signal series. It is unimaginable to consider that proteins would evolve a means to generate phonons without a means to route them.

If time is an essential component in non-linear events [25], it should be noted that others report such distant site activations are tightly tied to protein conformational changes [51, 52], and sequential ligand relaxation times [51]. Additionally, others [33, 42] corroborate Anfinsen's 1972 Nobel Prize findings that protein function is highly dependent on $\mathrm{pH}$ to speed or slow the process. For decades "classicist" argument has refuted non-linearity in cell signaling, however the iteration of quantized phonon signal series is a non-linear process http://www.phy.ilstu.edu/ ren/phononsims/page3.html). In combination with the other inter-dependent variables it would appear a classic linear solution is an antiquated proposal.

\section{ELECTRO-POLLUTION; NON-ELEMENTARY VIBRATIONS}

Based upon Curie's Law, $(\mathrm{M}=\mathrm{k} \mathrm{B} / \mathrm{T})$, if the imposed EM field (B) saturates PDC dipole alignment sufficiently, which need not be complete, their "damper" ability is compromised. In such an event it would seem possible to introduce extrinsic, "non-elementary modes" to cause structural or functional failure of "the system" if incompatible with the protein lattice's ability to absorb them. Outcomes possible when a harmonic forcer drives a sys- 
tem are: 1 . the system changes design, e.g. a swing angle, 2. energy output must equal energy input, 3 . the system fails. As noted in Fundamentals In Physics, [26], "care must be taken not to subject a system to a strong external driving force ... or the resulting oscillations may rupture it". Item 2 suggests that when heat energy in exceeds energy out the protein may be denatured resulting in a functional failure. The non-elementary vibrations associated with an EM force capable of saturating dipole activity and nullifying damping by PDCs are proposed as such oscillations. DNA due to its size, design, and quantity of electromagnetic responsive constructs may be quintessentially vulnerable to non-elementary resonant energy.

Elementary vs non-elementary dynamics must be considered when "lumping" therapeutic and "electro-pollution" field effects since one is designed to be native to the protein and the others non-elementary energy that must follow outcomes two or three. Switching large field strengths create field gradients that strongly argue against a "more is better" approach in DEPTH applications, e.g. rTMS and treatment of depression.

The upside of non-elementary resonant vibration suggests its use to destroy infectious pathogens. HIV and other pathogens undergo vulnerable dynamic adaptations, e.g. g41 fusion protein, that promise compromised function or structure with appropriate vibrational exposure Successful eradication of Plasmodium falciparum in Ghana after a single DEPTH treatment has been reported [22]; that report cites a different vibrational mechanism than is suggested here.

Trial and error will be necessary to find non-elementary vibrational modes necessary to degrade the function or structure of vulnerable proteins. Anecdotal observations over 25 yrs on treating infectious conditions with DEPTH, e.g. fistuli, otitis, lymphangitis, suggests the originating EM field can be within therapeutic limits with outcomes that indicate a startlingly rapid functional or structural compromise of agent virulence, if not viability. Satisfied with the rapid dehiscence of pain and inflammation, one overlooked the obvious implication in permanently improved outcomes that required no additional treatment.

Toxic metals and chemical toxins may substitute very different energy into PDC bonds, which then oscillate at very different output modes than the original construct to create a "non-elementary" response to an otherwise normal EM force, perhaps the long-term lethal threat in such bond substitutions.

\section{ELECTROGENOMICS}

The use of designed EM pulses to up-regulate DNA received substantial support when the Columbia University group headed by Blank and Goodman reported that site-specific electromagnetic responsive elements (EMREs) regulate DNA synthesis $[11,40]$. In spite of their observation these authors propose other than paramagnetic/diamagnetic dynamics, signal series, and conformational adaptation of DNA to activate gene loci. It could be proposed that electron transit across weak hydrogen bonds between base amino acid pairs doesn't occur "de novo", which suggests such enthalpy is an iterative, conformational adaptive activity. This same group reported that up-regulation was increased when transduced via multiple, similar EMRE constructs vs. just one or two [40], which strongly suggests an EMF/PDC/phonon iteration effect as the first order mechanism. [6, 18, 44]

\section{CALCIUM AND $\mathrm{Ca}^{++}$CHANNEL ACTIVITY}

Eichwald and Walleczek [21] reported the biological-functional status of treated tissue can result in EMF stimulation, inhibition, or no effect, which they attributed to "activation of specific EMF sensitive enzyme systems that modulate calcium entry." Calcium is an alkaline earth metal, diamagnetic, and considered "very reactive" [56]; it and water are both diamagnetic and highly mobile in EM fields, which along cell membranes with their myriad PDCs can only be imagined. Without noting its diamagnetic nature, calcium flux in response to EM fields was reported [39], and Dihel et al. [29] demonstrated such observations were related to Ca channel activity. Davies and Norris [19] demonstrated that $\mathrm{Ca}++$ dependent motility in marine diatoms, was substantially enhanced by EMFs. Using pheochromocytoma cells, Ikehara et al.[28] demonstrated EMFs inhibit increases in cytosolic $[\mathrm{Ca}++]$ by limiting release from intracellular stores, which was rapid (15 min) and lasted for two hours in the face of unchanged ATP levels over that period. Gibbs et al demonstrated cysteine rich secretory protein domains regulate ion channel activity, "and provide compelling evidence for a role in $[\mathrm{Ca}++]$ regulation..." Cysteine is proposed a classic PDC.

Vendel et al [54] reported, "Ca++ channel beta sub-units regulate trafficking and gating of voltage dependent $\mathrm{Ca}++$ channel alpha subunits", indicating beta subunits are the protein intelligence that computationally processes acoustic signal series. Using signal series to position voltage gated alpha subunits into appropriate position to achieve homeostasis is the precisely computed outcome of this "low voltage" information control circuit; given this direction, the parallel ATP driven energy system fulfills its "power circuit" role by appropriately moving substrate and solute to complete the homeostatic effort.

CPA (folding) has been demonstrated as a direct PDC effect [52], and Rosen's [47] reference to $\mathrm{Ca}++$ channel deformation from "anisotropic diamagnetic" phospolipids in the cell membrane might be expanded to incorporate many other PDCs [7], not to mention transitional metals. Vendel [53] further notes, "these domains that regulate cell surface expression and movement of $\mathrm{Ca}++$ voltage gated alpha sub-units involve five domains related to, "a large family of membrane-associated guanylate kinase proteins", conformational adaptation suggested to be a (phonon) signal series driven outcome. Mustafi et al. [43] report essentially similar findings in terms of multiple domains as part of "paramagnetic interactions with diamagnetic lanthanides, which substitute for $\mathrm{Ca}++$ ".

PDCs are major contributors to conformational protein 
control of $\mathrm{Ca}++$ channel activity, very probably $\mathrm{Ca}++$ routing through the channel, and cytosolic $[\mathrm{Ca}++]$ release [28]. Lastly, Baureus Koch et al. [8] studied weak EMF effects on the cell membrane and found, "suitable combinations of time varying magnetic fields directly interact with $\mathrm{Ca}++$ channel proteins in the cell membrane".

\section{CONCLUSION}

Adey noted [3], "Today we stand at a new, far more significant frontier, and while it may be more difficult to understand, it is at the atomic level rather than the molecular that physical rather than chemical processes shape the flow of signals essential to living matter ... one of the great revolutions in the history of biology". Adey was an MD who spent much of his career seeking the connection between cell function and the universal force of electromagnetism. This author proposes that PDC response to electromagnetic forcers, which has been overlooked for a century, keys the sophisticated information network proteins rely upon for conformational adaptive response $[1,2$, $9,41,45,46,52]$. Almost as an afterthought, redox reactions and ATP complete cell response.

It is apropos that we modify EB Wilson's 1950s quote, [4], "the key to every biologic problem must finally be sought in the cell" to that of a 2008 version, "must finally be sought in the physics of phonons, native low frequency vibrations that drive conformational protein adaptation", the cell will follow. Phonon physics and conformational protein adaptation are central to the paradigm beyond molecular physiology. Recent articles [6, 18] report DNA's use of noise to up-regulate gene synthesis and the sophisticated capacity of proteins to extract specific harmonics from overtones to enhance sub-threshold acoustic information. Electromagnetism both initiates and routes these vital phonon signals to control biological response; a sophisticated information system would have it no other way.

This understanding suggests overlooked avenues to control cell function, DNA up-regulation, and enzyme activity in response to ischemia-reperfion injury and other threats that will be better served by technologies as opposed to current drug regimens and numerous surgeries.

Proteins initiated this low energy conformational adaptive system upon first evolving, two or more billion years before the appearance of a nerve network, and still further removed from a central nervous system. A step toward returning to Rachel Carson's, "natural balance of things" and the human condition would be to enhance iterative protein activity rather than "blocking" or "inhibiting" it.

\section{REFERENCES}

[1] W.R. Adey. (1988) The cellular microenvironment and signaling through cell membranes. Progress in Clinical and Biological Research 257, 81-106.

[2] W.R. Adey. (1988) Cell Membranes: The electromagnetic environment and cancer promotion. Neurochemistry Research 7, 671-677.

[3] W.R. Adey. (1993) Biologic Effects of Electromagnetic Fields. Journal of Cellullar Biochemistry 4, 410-416.
[4] B. Alberts, D. Bray, J. Lewis, M. Raff, K. Roberts, J.D. Watson. (1989) Molecular Biology of The Cell, 2nd Ed. New York:Garland Publishing, preface.

[5] Angaridis P, Cotton FA, Murillo CA, Villagran D, Wang X. (2004). Paramagnetic precursors for supramolecular assemblies: selective syntheses, crystal structures, and electrochemical and magnetic properties of $\mathrm{Ru} 2(\mathrm{O} 2 \mathrm{CMe}) 4-\mathrm{n}$ (formamidinate) $\mathrm{nCl}$ complexes, $\mathrm{n}=$ 1-4. Inorganic Chemistry. Dec 26, 8290-8300.

[6] D.W. Austin, M.S. Allen, J.M. McCollum, R.D. Dar, J.R. Wilgus, G.S. Sayler, N.F. Samatova, C.D. Cox, M.L. Simpson. (2006) Gene network shaping of inherent noise spectra. Nature 7076, 608-611.

[7] S. Balakrishnan, N.J. Zondlo. (2006) Design of a protein kinase-inducible domain. Journal of the American Chemical Society 17, 5590-5591.

[8] C.L. Baureus Koch, M. Sommarin, B.R. Persson, L.G. Salford, J.L. Eberhardt. (2003) Interaction between weak low frequency magnetic fields and cell membranes. Bioelectromagnetics 6, 395-402.

[9] R.O. Becker,. Exploring new horizons in electromedicine. Journal Alternative and Complementary Medicine 1, 17-18.

[10] I. Bertini, A. Donaire, B. Jimenez, C. Luchinat, G. Parigi, M. Piccioli, L. Poggi. (2001) Paramagnetism-based versus classical constraints: an analysis of the solution structure of Ca Ln calbindin D9k. Journal of Biomolecular NMR 2, 85-98.

[11] M. Blank, R. Goodman. (2004) Initial interactions in electromagnetic field-induced interactions. Journal of Cellular Physiology 3, 359-363.

[12] S. Carmody, X.L. Wu, H. Lin, M. Blank, R. Goodman (2000) Cytoprotection by electromagnetic field-induced hsp70: a model for clinical appl;ication. J Cellular Biochem, 79: 453-459.

[13] K.C. Chou. (1984) Low-frequency vibration of DNA molecules. Biochemical Journal 221, 27-31.

[14] K.C. Chou. (1985) Low-frequency motions in protein molecules: beta-sheet and beta-barrel. Biophysical Journal 48, 289-297.

[15] K.C. Chou. (1988) Review: Low-frequency collective motion in biomacromolecules and its biological functions. Biophysical Chemistry 30, 3-48.

[16] K.C. Chou. (1989) Low-frequency resonance and cooperativity of hemoglobin. Trends in Biochemical Sciences 14, 212.

[17] K.C. Chou, and N.Y. Chen. (1977) The biological functions of low-frequency phonons. Scientia Sinica 20, 447-457.

[18] C.D. Cox, J.M. McCollum, D.W. Austin, M.S. Allen, R.D. Dar, M.L. Simpson. (2006) Frequency domain analysis of noise in simple gene circuits. Chaos 16, 26102.

[19] M.S. Davies, W.T. Norris. (2004). Vibration as a possible explanation for putative electromagnetic field effects: a case study on marine diatoms. International Journal of Radiation Biology10, 709-718.

[20] L.E. Dihel, J. Smith-Sonneborn, C.R. Middaugh. (1985) Effects of an extremely low frequency electromagnetic field on the cell division rate and plasma membrane of Paramecium tetraurelia. Bioelectromagnetics $1,61-71$.

[21] C. Eichwald, J. Walleczek. (1996) Activation-dependent and biphasic electromagnetic field effects: model based on cooperative enzyme kinetics in cellular signaling. Bioelectromagnetics 6, 427-435.

[22] J.E. Feagin, M.A. Wurschler, R. Ceon, H.C. Lai. (1999) Magnetic Fields and Malaria, "Biologic Effects of Light: Proceedings of the Biologic Effects of Light Symposium". Holick, M.F. and Jung E.G. (eds). Kluwer Academic Publishers, Higham MA, 343-349.

[23] G.M. Gibbs, M.J. Scanlon, J. Swarbrick, S. Curtis, E. Gallant, A.F. Dulhunty, M.K. O'Bryan. (2006) The cysteine-rich secretory protein domain of Tpx-1 is related to ion channel toxins and regulates ryanodine receptor $\mathrm{Ca} 2+$ signaling. $\mathrm{J}$ Biological Chemistry 7, 4156-4163.

[24] G.A. Gordon. (2007) Designed Electromagnetic Pulsed Therapy: Clinical Uses. J Cellular Physiology, 579-582.

[25] S. Hawking. (1988) A Brief History of Time. New York: Bantam Books. p. 61.

[26] D. Halliday, R. Resnick, J. Walker. (1993) Fundamentals of Physics, $4^{\text {th }}$ Ed. New York City: John Wiley \& Sons Inc., 399-400.

[27] A.R. Holzwarth, M.G. Muller. (1996). Energetics and kinetics of radical pairs in reaction centers from Phodobacter sphaeroides. A femtosecond transient study. Bioechemistry 36, 11820-11831.

[28] T. Ikehara, H. Yamaguchi, K. Hosokawa, H. Houchi, K.H. Park, K. 
Minakuchi, H. Kashimoto, M. Kitamura, Y. Kinouchi, K. Yoshizaki, H. Miyamoto. (2005) Effects of a time-varying strong magnetic field on transient increase in $\mathrm{Ca} 2+$ release induced by cytosolic $\mathrm{Ca} 2+$ in cultured pheochromocytoma cells. Biochemica Biophysica Acta 1-2, 8-16.

[29] J. Iwahara, G.M. Clore. (2006) Detecting transient intermediates in macromolecular binding by NMR. Nature 7088, 1227-1230.

[30] M.K. Johnson, J.E. Morningstar, M. Oliver, F.E. Frerman. (1987) Electron paramagnetic resonance and magnetic circular dichroism studies of electron-transfer flavoprotein-ubiquinone oxireductase from pig liver. FEBS Letters 1, 129-133.

[31] M.T. Johnson, L.R. Waite, G. Nindl. (2004) Non-invasive treatment of inflammation using EM fields: current and emerging therapeutic potential. Biomedical Sciences Instrumentation 40, 469-474.

[32] G.J. Kornhaber, D. Snyder, H.N. Moseley, G.T. Montelione. (2006) Identification of zinc-ligated cysteine residues based on 13Calpha and 13Cbeta chemical shift data. J Biomolecular NMR 4, 259-269.

[33] J.M. Kriegl, A.J. Bhattacharyya, K. Nienhaus, P. Deng, O. Minkow, G.U. Nienhaus. (2002) Ligand binding and protein dynamics in neuroglobin. Proceedings of the National Academy of Sciences, USA 12, 7992-7997.

[34] J.M. Kriegl, G.U. Niehaus. (2004) Structural, dynamic, and energetic aspects of long-range electron transfer in photosynthetic reaction centers. Proceedings of The National Academy of Sciences, USA 1, 12312-12318.

[35] I.L. Kruglikov, H. Dertinger.(1994) Stochastic resonance as a possible mechanism of amplification of weak electric signals in living cells. Bioelectromagnetics 6, 539-547.

[36] A.F. Lawrence, W.R. Adey. (1982). Nonlinear wave mechanisms in interactions between excitable tissue and electromagnetic fields. Neurological Research 4, 115-153.

[37] A.F. Lawrence et al. (1987) The Nature of Phonons and Soliton Waves in Alpha Helical Proteins. Journal of Biophysics 51-5, 785-93.

[38] A. Liboff. (2004) Toward an electromagnetic paradigm for biology and medicine, Journal Alternative and Complementary Medicine 1, 41-47.

[39] R.J. Lieb, W. Regelson, B. West, R.L. Jordan, D.P. DePaola. (1980) Effect of pulsed high frequency electromagnetic radiation on embryonic mouse tissue palate in vitro. Journal of Dental Research 10, 1649-1652.

[40] H. Lin, M. Blank, K. Rossol-Haseroth, R. Goodman. (2001) Regulating genes with electromagnetic response elements. Journal of Cellular Biochemistry 1, 143-148.

[41] B.R. McLeod, A.R. Liboff, S.D. Smith. (1992) Electromagnetic gating in ion channels. Journal of Theoretical Biology 1, 15-31.

[42] O.B. Morozova, S.E. Korchak, R.Z. Sagdeev, A.V. Yurkovskaya. (2005) Time-resolved chemically induced dynamic nuclear polarization studies of structure and reactivity of methionine radical cations in aqueous solution as a function of $\mathrm{pH}$. Journal of Physical Chemistry A 45, 10459-10466.

[43] S.M. Mustafi, S. Mukherjee, K.V. Chary, C. Del Bianco, C. Luchinat. (2004) Energetics and mechanism of $\mathrm{Ca} 2+$ displacement by lanthanides in a calcium binding protein. Biochemistry 29, 9320-9331.

[44] J.M. Pedraza, A. van Oudenaarden. (2005) Noise propagation in gene networks. Science 5717, 1965-1969.

[45] B. Rabenstein, G.M. Ullmann, E.W. Knapp. (2000) Electron transfer between the quinones in the photosynthetic reaction center and its coupling to conformational changes. Biochemistry 34, 10487-10496.

[46] V. Ramikrishnan, D. Henderson, D.D. Busath. (2004) Applied field nonequilibrium molecular dynamics simulations of ion exit from a beta-barrel model of the L-type calcium channel. Biochimica et Biophysica Acta 1, 1-8.

[47] A.D. Rosen. (2003). Mechanism of action of moderate-intensity static magnetic fields on biological systems. Cell Biochemistry and Biophysics 2, 163-174

[48] A. Schenle, V. Starkand. (1998) J Scientific Exploration 12, 455-468.

[49] H.M. Sobell, C.C. Tsai, S.C. Jain, T.D. Sakore. (1978) Conformational flexibility in DNA structure and its implication in understanding the organization of DNA in chromatin. Philos. Trans. R. Lond. B. Biol. Sci. 11, 295-298.

[50] C. Strohm, G..L. Rikken, P. Wyder. (2005) Phenomenologic Evidence for the Phonon Hall Effect. Phys Rev Lett 95, 155901, 2005.

[51] J. Tandori, P. Sebban, H. Michel, L. Baciou. (1999) In Rhodobacter sphaeroides reaction centers, mutation of proline L209 to aromatic residues in the vicinity of a water channel alters the dynamic coupling between electron and proton transfer processes. Biochemistry 40, 13179-13187.

[52] M. Ubbink, M. Ejdeback, B.G. Karlsson, D.S. Bendall. (1998) The structure of the complex of plastocyanin and cytochrome f, determined by paramagnetic NMR and restrained rigid-body molecular dynamics. Structure 3, 323-335.

[53] A.C. Vendel, C.D. Rithner, B.A. Lyons, W.A. Horne. (2006) Solution structure of the N-terminal A domain of the human voltage-gated $\mathrm{Ca} 2+$ channel beta4a subunit. Protein Science 2, 378-383.

[54] A.C. Vendel, M.D. Terry, A.R. Striegel, N.M. Iverson, V. Leuranguer, C.D. Rithner.

a) Voltage-gated $\mathrm{Ca} 2+$ channel beta4 subunit creates a uniquely folded

b) N-terminal protein binding domain with cell-specific expression in the c) cerebellar cortex. Journal of Neuroscience10, 2635-2644.

[55] M. Wang, R.T. Borchardt, R.L. Schowen, K. Kuczera. (2005) Domain motion and the open-to-closed conformational transition of an enzyme: a normal mode analysis ... Biochemistry 17, 7228-7239.

[56] S. Zumdahl. (1992) Chemical Principles. Lexington MA: DC Heath and Co. p 980. 